\title{
DOI: https://doi.org/10.24297/jap.v16i1.8375
}

\section{Comparison Between Thermo Luminescence Dosimeter and Planning System Dose Calculation in The Brain and Spinal Cord Tumour}

\author{
Ehab A Hegazy \\ Delta University for science and technology, Mansoura, Egypt. \\ Ehab.hegazy@deltauniv.edu.eg, Ehabhegazy99@yahoo.com
}

\begin{abstract}
Radiotherapy of Spinal cord and brain tumor requires High care due to considerable changes in the white matter of the brain, which consequently lead to a reduction of patient learning and mental skills. It is considered a very critical tumor due to high sensitivity of gross volume location and normal tissues surrounding it, including eye, heart, plate thyroid, and testis. XiO planning systems, TLD dosimeter found in Mansoura university oncology department, CMS XIO USA TPS were compared using electron and photon beams with different energies at a different site in target volume and organs at risk. We conclude that regular calibration of planning systems and direct measurement of the dose delivered to main target and organs at risk should be done to avoid the difference between XiO planning systems and direct measurement by TLd.
\end{abstract}

Keywords: Spinal cord, radiotherapy, luminescence

\section{Introduction}

Brain and spinal cord tumor are widely spread all over the word, it is considered as very critical tumor due to high sensitivity of gross volume location and normal tissues surrounding it including eye, heart, plate thyroid and testis [1]. High care should be taken due to considerable changes in the white matter of the brain, which consequently lead to a reduction of patient learning and mental skills [2].

Calculation of dose in different patient organs using the basic ideas of photon interactions with tissue alone is inaccurate depends on direct measurements by phantoms physicist derived the basic dose quantities calculations [3]. To reach the optimal dose determination, multiple beams from 7 to 13 field should be used to maximize the dose delivered to the target, and protecting the critical structures $[4,5]$.

TLDs dosimeter is considered an accurate method for detecting radiation dose as their active volume can be practically designed very small as compared to the ionization chamber and other devices [6]. Its operation depends on oven temperature control [7]. Thermo-luminescence physical phenomena can be explained by the energy band theory of solids [8].

To simulate the actual treatment conditions and parameter, physicists use the treatment planning systems (TPSs) [9]. These systems use algorithms to calculate doses for tumor and other organs. [10]. The accuracy of dose calculation is very important to make sure that the calculated dose is similar to the dose received by the organ Aim of quality assurance of TPS is to be sure that the dose is calculated accurately [11].

Experimental dose measurements are usually performed using simple rectangular or cylindrical phantoms with various detectors (e.g., an ionization chamber or radiochromic film) [12-14].

Present study aimed to evaluate the accuracy of using CMS XiO TPS in the brain and spinal cord tumor radiotherapy and using two different craniospinal radiotherapy techniques. A comparison between dose measured by TLs technique and those calculated by TBS is done. 


\section{Materials and Methods}

CT diagnostic unit was manufactured by Siemens model SOMATOM sensation performance in Germany was used in this study. It is used to generate an image of the inside of an object from a large series of twodimensional images.

Symptom sensation CT unit was used in this study to form an image of the target tumor in 2 D model. TLD 100 harshow Cleveland H USA and prorad Germany were distributed equally at different location interiorly for photon and electron. The results obtained by TLD were compared by those of CMS XIO USA TPS. Organs at risk were carefully contorted by physician. Linear accelerator $6 \mathrm{mv}$ was used type eleckta precise. Ionization chamber type farmer was used as calibration method and for quality assurance.

\section{Results and Discussion}

Tables from 1 to 5 show the difference between the direct reading of TLD and planning systems for target tumor and organs at risk for photon and electron beam.

Table 1: difference in thyroid dose for electron beam at energy 6 mev and 18 mev and photon 6 and 15 mev measured by TLD and planning system XiO.

\begin{tabular}{llll}
\hline & Electron field & \multicolumn{2}{l}{ Photon field } \\
\hline 1.1 & 4.63 & -5.24 & -1.15 \\
\hline 1.4 & 4.93 & -4.94 & -0.85 \\
1.7 & 5.23 & -4.64 & -0.55 \\
\hline 2 & 5.53 & -4.34 & -0.25 \\
\hline 2.3 & 5.83 & -4.04 & 0.05 \\
\hline 2.6 & 6.13 & -3.74 & 0.35 \\
\hline 2.9 & 6.43 & -3.44 & 0.65 \\
\hline 3.2 & 6.73 & -3.14 & 0.95 \\
\hline 3.5 & 7.03 & -2.84 & 1.25 \\
\hline 3.8 & 7.33 & -2.54 & 1.55 \\
\hline 4.1 & 7.63 & -2.24 & 1.85 \\
\hline 4.4 & 7.93 & -1.94 & 4.25 \\
\hline 4.7 & 10.23 & -1.64 & 2.45 \\
\hline 5 & 8.53 & -1.34 & 2.75 \\
\hline 5.3 & 8.83 & -1.04 & 3.05 \\
\hline 5.6 & 9.13 & -0.74 & 3.35 \\
\hline 5.9 & 9.43 & -0.44 & 3.65 \\
\hline 6.2 & 9.73 & -0.14 & 3.95 \\
\hline 6.5 & 10.03 & 0.16 & 4.25 \\
\hline
\end{tabular}


For thyroid gland, which is considered as an organ at risk. The maximum difference for electron beam was 10.23 $\%$, and for photon beam was 5.24 , this means that accurate care this may be due to the small size of the thyroid gland.

Table 2: Difference in heart dose for electron beam at energy 6 mev and 18 mev and photon 6 and 15 mev measured by TLD and planning system XiO.

\begin{tabular}{|c|c|c|c|}
\hline \multicolumn{2}{|c|}{ Electron Field } & \multicolumn{2}{|c|}{ Photon Field } \\
\hline 1.7 & 5.23 & -4.64 & -0.55 \\
\hline 1.9 & 5.43 & -4.44 & -0.35 \\
\hline 2.1 & 5.63 & -4.24 & -0.15 \\
\hline 2.3 & 5.83 & -4.04 & 0.05 \\
\hline 2.5 & 6.03 & -3.84 & 0.25 \\
\hline 2.7 & 6.23 & -3.64 & 0.45 \\
\hline 2.9 & 6.43 & -3.44 & 0.65 \\
\hline 3.1 & 6.63 & -3.24 & 0.85 \\
\hline 3.3 & 6.83 & -3.04 & 1.05 \\
\hline 3.5 & 7.03 & -2.84 & 1.25 \\
\hline 3.7 & 7.23 & -2.64 & 1.45 \\
\hline 3.9 & 7.43 & -2.44 & 1.65 \\
\hline 4.1 & 7.63 & -2.24 & 1.85 \\
\hline 4.3 & 7.83 & -2.04 & 2.05 \\
\hline 4.5 & 8.03 & -1.84 & 2.25 \\
\hline 4.7 & 8.23 & -1.64 & 2.45 \\
\hline 4.9 & 8.43 & -1.44 & 2.65 \\
\hline 5.1 & 8.63 & -4.70 & 2.85 \\
\hline 5.3 & 8.83 & -1.04 & 3.05 \\
\hline 5.5 & 9.03 & -0.84 & 3.25 \\
\hline 5.7 & 9.23 & -0.64 & 3.45 \\
\hline
\end{tabular}


For heart which is considered as an organ at risk. The maximum difference for electron beam was 9.23, and for photon beam was $4.70 \%$ this might be due to the movement of the heart muscle.

Table 3: difference in brain and spinal cord dose for electron beam at energy 6 mev and 18 mev and photon 6 and 15 mev measured by TLD and planning system XiO.

\begin{tabular}{|c|c|c|c|c|}
\hline Electron Beam & & Photon Beam & & \\
\hline-2.59 & -3.45 & -1.58 & & -1.25 \\
\hline-1.49 & -2.35 & -0.48 & -0.15 & \\
\hline-0.39 & -1.25 & 0.62 & 0.95 & \\
\hline 0.71 & -0.15 & 1.72 & 2.05 & \\
\hline 1.81 & 0.95 & 2.82 & 3.15 & \\
\hline 2.91 & 2.05 & 3.92 & 4.25 & \\
\hline 4.01 & 3.15 & 5.02 & 5.35 & \\
\hline 5.11 & 4.25 & 6.12 & 6.45 & \\
\hline 6.21 & 5.35 & 7.22 & 7.55 & \\
\hline 7.31 & 6.45 & 8.32 & 8.65 & \\
\hline 8.41 & 7.55 & 9.42 & 9.75 & \\
\hline 9.51 & 8.65 & 10.52 & 10.85 & \\
\hline 10.61 & 9.75 & 11.62 & 11.95 & \\
\hline 11.71 & 10.85 & 12.72 & 13.05 & \\
\hline 12.81 & 11.95 & 13.82 & 14.15 & \\
\hline 13.91 & 13.05 & 14.92 & 15.25 & \\
\hline 15.01 & 14.15 & 16.02 & 16.35 & \\
\hline 16.11 & 15.25 & 17.12 & 17.45 & \\
\hline 17.21 & 16.35 & 18.22 & 18.55 & \\
\hline 18.31 & 17.45 & 19.32 & 19.65 & \\
\hline 19.41 & 18.55 & 20.42 & 20.75 & \\
\hline 20.51 & 19.65 & 21.52 & 21.85 & \\
\hline 21.61 & 20.75 & 22.62 & 22.95 & \\
\hline 22.71 & 21.85 & 23.72 & 24.05 & \\
\hline 23.81 & 22.95 & 24.82 & 25.15 & \\
\hline
\end{tabular}

For target volume brain and spinal cord, which are the main issues for this study.The maximum difference for electron beam was $23.81 \%$ and for photon beam was 25.15 this difference is significantly high.

Table 3: Difference in left and right lung for electron beam at energy 6 mev and 18 mev and photon 6 and 15 
mev measured by TLD and planning system XiO.

\section{Left lung}

Right lung

\begin{tabular}{|c|c|c|c|c|c|c|c|}
\hline 0.79 & 1.64 & 0.74 & 1.56 & -1.03 & 1.71 & -1.87 & 2.54 \\
\hline 0.99 & 1.84 & 0.94 & 1.76 & -0.83 & 1.91 & -1.67 & 2.74 \\
\hline 1.19 & 2.04 & 1.14 & 1.96 & -0.63 & 2.11 & -1.47 & 2.94 \\
\hline 1.39 & 2.24 & 1.34 & 2.16 & -0.43 & 2.31 & -1.27 & 3.14 \\
\hline 1.59 & 2.44 & 1.54 & 2.36 & -0.23 & 2.51 & -1.07 & 3.34 \\
\hline 1.79 & 2.64 & 1.74 & 2.56 & -0.03 & 2.71 & -0.87 & 3.54 \\
\hline 1.99 & 2.84 & 1.94 & 2.76 & 0.17 & 2.91 & -0.67 & 3.74 \\
\hline 2.19 & 3.04 & 2.14 & 2.96 & 0.37 & 3.11 & -0.47 & 3.94 \\
\hline 2.39 & 3.24 & 2.34 & 3.16 & 0.57 & 3.31 & -0.27 & 4.14 \\
\hline 2.59 & 3.44 & 2.54 & 3.36 & 0.77 & 3.51 & -0.07 & 4.34 \\
\hline 2.79 & 3.64 & 2.74 & 3.56 & 0.97 & 3.71 & 0.13 & 4.54 \\
\hline 2.99 & 3.84 & 2.94 & 3.76 & 1.17 & 3.91 & 0.33 & 4.74 \\
\hline 3.19 & 4.04 & 3.14 & 3.96 & 1.37 & 4.11 & 0.53 & 4.94 \\
\hline 3.39 & 4.24 & 3.34 & 4.16 & 1.57 & 4.31 & 0.73 & 5.14 \\
\hline 3.59 & 4.44 & 3.54 & 4.36 & 1.77 & 4.51 & 0.93 & 5.34 \\
\hline 3.79 & 4.64 & 3.74 & 4.56 & 1.97 & 4.71 & 1.13 & 5.54 \\
\hline 3.99 & 4.84 & 3.94 & 4.76 & 2.17 & 4.91 & 1.33 & 5.74 \\
\hline 4.19 & 5.04 & 4.14 & 4.96 & 2.37 & 5.11 & 1.53 & 5.94 \\
\hline 4.39 & 5.24 & 4.34 & 5.16 & 2.57 & 5.31 & 1.73 & 6.14 \\
\hline 4.59 & 5.44 & 4.54 & 5.36 & 2.77 & 5.51 & 1.93 & 6.34 \\
\hline 4.79 & 5.64 & 4.74 & 5.56 & 2.97 & 5.71 & 2.13 & 6.54 \\
\hline 4.99 & 5.84 & 4.94 & 5.76 & 3.17 & 5.91 & 2.33 & 6.74 \\
\hline 5.19 & 6.04 & 5.14 & 5.96 & 3.37 & 6.11 & 2.53 & 6.94 \\
\hline
\end{tabular}

For organs at risk lungs show relatively small differences as follows, the maximum difference was $6.04 \%$, and for the right lungs, the difference was $6.94 \%$. 
Table 4 : Percentage difference between the results of thermo luminescent dosimeter and treatment planning system for measurement points inside the photon treatment field.

\begin{tabular}{|l|l|l|}
\hline$\underline{\underline{C T V}}$ & $\underline{\text { Chiasma }}$ & Mandible \\
\hline-1.58 & -1.55 & $\mathbf{0 . 2 1}$ \\
\hline-0.58 & 1.53 & $\mathbf{2 . 2 5}$ \\
\hline 0.51 & -0.27 & $\mathbf{- 1 . 2 7}$ \\
\hline-2.47 & & $\mathbf{0 . 8 1}$ \\
\hline 0.24 & -0.34 & $\mathbf{3 . 8 8}$ \\
\hline-0.44 & & $\mathbf{2 . 4 2}$ \\
\hline-0.78 & & 3.73 \\
\hline
\end{tabular}

For organs at risk chiams and mandible which are very close to the brain and spinal cord differences are very variable as shown in table 4 , the measurement was taken inside and outside the organs, and $3.88 \%$ was the maximum difference observed.

Table 5: Difference in left and right kidney for electron beam at energy 6 mev and 18 mev and photon 6 and 15 mev measured by TLD and planning system XiO.

\begin{tabular}{|c|c|c|c|c|c|c|c|}
\hline \multicolumn{4}{|c|}{ Left kidney } & \multicolumn{4}{|c|}{ Right kidney } \\
\hline \multicolumn{2}{|c|}{ Electron beam } & \multicolumn{2}{|c|}{ Photon beam } & \multicolumn{2}{|c|}{ Electron beam } & \multicolumn{2}{|c|}{ Photon beam } \\
\hline 2.11 & 0.3 & 4.77 & 1 & 2.24 & 0.61 & 3.88 & 1.1 \\
\hline 2.22 & 0.41 & 4.88 & 1.11 & 2.35 & 0.72 & 3.99 & 1.21 \\
\hline 2.33 & 0.52 & 4.99 & 1.22 & 2.46 & 0.83 & 4.1 & 1.32 \\
\hline 2.44 & 0.63 & 5.1 & 1.33 & 2.57 & 0.94 & 4.21 & 1.43 \\
\hline 2.55 & 0.74 & 5.21 & 1.44 & 2.68 & 1.05 & 4.32 & 1.54 \\
\hline 2.66 & 0.85 & 5.32 & 1.55 & 2.79 & 1.16 & 4.43 & 1.65 \\
\hline 2.77 & 0.96 & 5.43 & 1.66 & 2.9 & 1.27 & 4.54 & 1.76 \\
\hline 2.88 & 1.07 & 5.54 & 1.77 & 3.01 & 1.38 & 4.65 & 1.87 \\
\hline 2.99 & 1.18 & 5.65 & 1.88 & 3.12 & 1.49 & 4.76 & 1.98 \\
\hline 3.1 & 1.29 & 5.76 & 1.99 & 3.23 & 1.6 & 4.87 & 2.09 \\
\hline 3.21 & 1.4 & 5.87 & 2.1 & 3.34 & 1.71 & 4.98 & 2.2 \\
\hline 3.32 & 1.51 & 5.98 & 2.21 & 3.45 & 1.82 & 5.09 & 2.31 \\
\hline 3.43 & 1.62 & 6.09 & 2.32 & 3.56 & 1.93 & 5.2 & 2.42 \\
\hline 3.54 & 1.73 & 6.2 & 2.43 & 3.67 & 2.04 & 5.31 & 2.53 \\
\hline 3.65 & 1.84 & 6.31 & 2.54 & 3.78 & 2.15 & 5.42 & 2.64 \\
\hline 3.76 & 1.95 & 6.42 & 2.65 & 3.89 & 2.26 & 5.53 & 2.75 \\
\hline
\end{tabular}




\begin{tabular}{|l|l|l|l|l|l|l|l|l|}
\hline 3.87 & 2.06 & 6.53 & 2.76 & 4 & 2.37 & 5.64 & 2.86 \\
\hline 3.98 & 2.17 & 6.64 & 2.87 & & 4.11 & 2.48 & 5.75 & 2.97 \\
\hline 4.09 & 2.28 & 6.75 & 2.98 \\
4.2 & 2.39 & 6.86 & 3.09 & 4.22 & 2.59 & 5.86 & 3.08 \\
\hline 4.31 & 2.5 & 6.97 & 3.2 & 4.33 & 2.7 & 5.97 & 3.19 \\
\hline 4.42 & 2.61 & 7.08 & 3.31 & 4.44 & 2.81 & 6.08 & 3.3 \\
\hline 4.53 & 2.72 & 7.19 & 3.42 & 4.55 & 2.92 & 6.19 & 3.41 \\
\hline 4.64 & 2.83 & 7.3 & 3.53 & 4.66 & 3.03 & 6.3 & 3.52 \\
\hline $4 n$ & 4.75 & 2.94 & 7.41 & 3.64 & 4.77 & 3.14 & 6.41 & 3.63 \\
\cline { 6 - 7 } & & 4.88 & 3.25 & 6.52 & 3.74 \\
\hline
\end{tabular}

For the left and right kidney, which are close to the lower part of the spinal cord. The maximum difference for electron beam was $4.88 \%$ and for photon beam was $7.41 \%$ this difference is significantly high.

\section{Conclusion}

In this study, direct measurement of dose in the spinal cord and brain was accurately detected in order to measure the validity of using $\mathrm{XiO}$ treatment system like a dose specular detector. The significant difference was found in treatment dose delivered to the thyroid by the electron. This require high care for planning and calculation of the dose of electron treated thyroid gland. A large difference was detected in the heart, but controversy to thyroid heart is radio resistant and can recover over dose to radiation, care should be taken for young age patient which may be subjected to heat failure in the next 10 years. In other organs, the difference is less than $10 \%$. We conclude that regular calibration of planning systems and direct measurement of the dose delivered to main target and organs at risk should be done to avoid the difference between XiO planning systems and direct measurement by TLd.

\section{References}

1. Spirou, S., Basini, M., Lascialfari, A., Sangregorio, C., \& Innocenti, C. (2018). Magnetic hyperthermia and radiation therapy: Radiobiological principles and current practice. Nanomaterials, 8(6), 401.

2. Mulhern, R. K., Palmer, S. L., Reddick, W. E., Glass, J. O., Kun, L. E., Taylor, J.,\& Gajjar, A. (2001). Risks of a young age for selected neurocognitive deficits in medulloblastoma are associated with white matter loss. Journal of Clinical Oncology, 19(2), 472-479.

3. Hegazy EA. Study of output difference of two different ionization chambers for large fields used in radiotherapy.JOURNAL OF ADVANCES IN PHYSICS. 2017 Jun 1;13(5):4901-7.

4. Han, K., Cheung, P., Basran, P. S., Poon, I., Yeung, L., \& Lochray, F. (2010). A comparison of two immobilization systems for stereotactic body radiation therapy of lung tumors. Radiotherapy and Oncology, 95(1), 103-108.

5. Brock, J., Bedford, J., Partridge, M., McDonald, F., Ashley, S., McNair, H. A., \& Brada, M. (2012). Optimizing stereotactic body radiotherapy for non-small cell lung cancer with volumetric intensity-modulated arc therapy—a planning study. Clinical Oncology, 24(1), 68-75.

6. Olko, P. (2010). Advantages and disadvantages of luminescence dosimetry. Radiation Measurements, 45(36), 506-511.

7. Low, D. A., Moran, J. M., Dempsey, J. F., Dong, L., \& Oldham, M. (2011). Dosimetry tools and techniques for IMRT. Medical Physics, 38(3), 1313-1338.

8. Siddique, R., Uddin, Z., \& Hussain, M. (2012). Dosimetric evaluation and verification of external beam 3-D treatment plans in humanoid phantom using thermo luminescent dosimeters (TLDs). Journal of Basic \& Applied Sciences, 8, 690-695. 
9. Mollazadeh, M., Allahverdi, M., Allahverdi Pourfallah, T., Riahi Alam, N., \& Ay, M. (2010). Evaluation of the RtDosePlan Treatment Planning System using Radiochromic Film and Monte Carlo Simulation. Iranian Journal of Medical Physics, 7(2), 81-93.

10. Lu, L. (2014). Dose calculation algorithms in external beam photon radiation therapy. International Journal of Cancer Therapy and Oncology, 1(2).

11. Koeck, J., Abo-Madyan, Y., Lohr, F., Stieler, F., Kriz, J., Mueller, R. P., ... \& Eich, H. T. (2012). Radiotherapy for early mediastinal Hodgkin lymphoma according to the German Hodgkin Study Group (GHSG): the roles of intensity-modulated radiotherapy and involved-node radiotherapy. International Journal of Radiation Oncology* Biology* Physics, 83(1), 268-276.

12. Ezzell, G. A., Burmeister, J. W., Dogan, N., LoSasso, T. J., Mechalakos, J. G., Mihailidis, D., ... \& Shi, J. (2009). IMRT commissioning: multiple institution planning and dosimetry comparisons, a report from AAPM Task Group 119. Medical Physics, 36(11), 5359-5373.

13. Zani, M., Bucciolini, M., Casati, M., Talamonti, C., Marinelli, M., Prestopino, G., ... \& Verona-Rinati, G. (2013). A synthetic diamond diode in volumetric modulated arc therapy dosimetry. Medical Physics, 40(9), 092103.

14. Kamomae, T., Oita, M., Hayashi, N., Sasaki, M., Aoyama, H., Oguchi, H., .. \& Naganawa, S. (2016). Characterization of stochastic noise and post-irradiation density growth for reflective-type radiochromic film in therapeutic photon beam dosimetry. Physica Medica, 32(10), 1314-1320. 